\title{
Computing Outer Inverses Using Complete Orthogonal Factorizations
}

\author{
Bilall I. Shaini ${ }^{1}$ \\ ${ }^{1}$ State University of Tetova, Rr. e Ilindenit, p.n., Tetovo, R. Macedonia \\ Correspondence: Bilall I. Shaini, State University of Tetova, Rr. e Ilindenit, p.n., Tetovo, R. Macedonia. E-mail: \\ bilall.shaini@unite.edu.mk
}

Received: May 16, 2014 Accepted: June 10, 2014 Online Published: August 4, 2014

doi:10.5539/jmr.v6n3p91～URL: http://dx.doi.org/10.5539/jmr.v6n3p91

\begin{abstract}
Several full-rank representations of the $A_{T, S}^{(2)}$ inverse of a given constant complex matrix, which are based on various complete orthogonal factorizations, are introduced. Particularly, we introduce a full rank representation based on the Singular Value Decomposition $(S V D)$ as well as on a combination of the $Q R$ decomposition and the $S V D$ of the triangular matrix produced by the $Q R$ decomposition. Furthermore, representations based on the factorizations to a bidiagonal form are defined. The representations arising from reductions to bidiagonal form are applicable to real full row rank matrices. Illustrative numerical examples as well as an extensive numerical study are presented. A comparison of three introduced methods is presented.
\end{abstract}

Keywords: generalized inverse $A_{T, S}^{(2)}$, complete orthogonal factorizations, $S V D$ factorization, $Q R$ factorization, full-rank representation

\section{Introduction}

Computation of generalized inverses by means of various matrix decompositions has been extensively investigated in the scientific literature.

A fast computational method for computing the Moore-Penrose inverse $A^{\dagger}$ based on the $Q R$ decomposition of the matrix $A$ is introduced in (Katsikis et al., 2011). The $Q R$ decomposition is assumed to be defined as in Theorem 3.3.11 in (Watkins, 2002) and its extension to complex matrices is used from (Godall, 1993). An extension of the representation introduced in (Katsikis et al., 2011) to the set of outer inverses with prescribed range and null space is presented in (Stanimirović et al., 2012b).

Symbolic computation of $A_{T, S}^{(2)}$ inverses on the basis of the $Q D R$ decomposition of the matrix $W$ is presented in (Stanimirović et al., 2012). The canonical form of the $D M P$ inverse $A^{D} A A^{\dagger}$, of a square matrix $A$, is presented in (Malik \& Thome, 2014). This representation of the DMP inverse is based on the Hartwig-Spindelböck matrix decomposition. The authors of the paper (Issa \& Bdair, 2014) introduced some new bounds for the zeros of polynomials by using the $Q R$ and $L U$ decompositions of the corresponding companion matrices. Recently, representations of $\{2,4\}$ and $\{2,3\}$ generalized inverses based on the $S V D$ are considered in (Shaini \& Hoxha, 2013).

In the present paper we develop several numerical algorithms for computing $A_{T, S}^{(2)}$ inverses. These algorithms are based on the full rank representation of an appropriately chosen $n \times m$ matrix $W$ arising from various complete orthogonal factorizations of $W$.

Our second intention is to examine properties of the introduced methods. For this reason, the paper presents a greater number of numerical experiments in which the introduced methods are compared with each other.

The paper is organized as follows. The second section surveys some useful basic notions and notations concerning generalized inverses. In the third section we derive two numerical algorithms for computing outer inverses. These algorithms are based on the $S V D$ factorization of an appropriately chosen matrix $W$ as well as on the successive application of $Q R$ decomposition and $S V D$. Representations based on bidiagonal forms, applicable to real full row rank matrices, are introduced in Section 4. Numerical examples on various test matrices are presented in the last section. 


\section{Preliminaries}

Following the usual notation, by $\mathbb{R}_{r}^{m \times n}$ (resp. $\mathbb{C}_{r}^{m \times n}$ ) we denote the set of all real (resp. complex) $m \times n$ matrices of rank $r$. By $I$ we denote the unit matrix of an appropriate order. Furthermore $A^{T}, \mathcal{R}(A), \operatorname{rank}(A)$ and $\mathcal{N}(A)$ denote the transpose, the range, the rank and the null space of $A \in \mathbb{R}^{m \times n}$.

For any matrix $A$ of the order $m \times n$ consider the following matrix equations in $X$, where $*$ denotes conjugate and transpose:
(1) $\quad A X A=A$
(2) $X A X=X$
(3) $(A X)^{*}=A X$
$(X A)^{*}=X A$.

In the case $m=n$ we also consider the following equations

$$
\text { (5) } A X=X A \quad\left(1^{k}\right) \quad A^{k+1} X=A^{k} \text {. }
$$

For a sequence $\mathcal{S}$ of elements from the set $\left\{1,2,3,4,5,1^{k}\right\}$, the set of matrices obeying the equations labeled by the numbers collected in $\mathcal{S}$ is denoted by $A\{\mathcal{S}\}$. A matrix from $A\{\mathcal{S}\}$ is called an $\mathcal{S}$-inverse of $A$. The matrix $X=A^{\dagger}$ is said to be the Moore-Penrose inverse of $A$ satisfying equations (1)-(4). The group inverse $A^{\#}$ is the unique $\{1,2,5\}$ inverse of $A$, and exists if and only if $\operatorname{ind}(A)=\min _{k}\left\{k \mid \operatorname{rank}\left(A^{k+1}\right)=\operatorname{rank}\left(A^{k}\right)\right\}=1$. A matrix $X=A^{D}$ is said to be the Drazin inverse of $A$ if $\left(1^{k}\right)$ (for some positive integer $k$ ), (2) and (5) are satisfied. In the case $\operatorname{ind}(A)=1$, the Drazin inverse of $A$ is equal to the group inverse $A^{\#}$ of $A$.

The rank of generalized inverse $X$ is important, and it will be convenient to consider the subset $A\{i, j, k\}_{s}$ of $A\{i, j, k\}$, consisting $\{i, j, k\}$-inverses of rank $s$ (see Ben-Israel \& Greville, 2003).

If $A \in \mathbb{R}_{r}^{m \times n}, T$ is a subspace of $\mathbb{R}^{n}$ of dimension $t \leq r$ and $S$ is a subspace of $\mathbb{R}^{m}$ of dimension $m-t$, then $A$ has a $\{2\}$-inverse $X$ such that $\mathcal{R}(X)=T$ and $\mathcal{N}(X)=S$ if and only if $A T \oplus S=\mathbb{R}^{m}$, in which case $X$ is unique and it is denoted by $A_{T, S}^{(2)}$. The outer generalized inverses with prescribed range and null-space are of the special importance in matrix theory. The $\{2\}$-inverses have application in constructing the iterative methods for solving the nonlinear equations (Ben-Israel \& Greville, 2003; Nashed, 1993) as well as in statistics (Getson \& Hsuan, 1988; Husen \& Langenberg, 1985). In particular, outer inverses play an important role in stable approximations of ill-posed problems and in linear and nonlinear problems involving rank-deficient generalized inverse (Nashed, 1976; Zheng \& Bapat, 2004). On the other hand, it is well known that the Moore-Penrose inverse $A^{\dagger}$ and the weighted Moore-Penrose inverse $A_{M, N}^{\dagger}$, the Drazin inverse $A^{D}$ and the group inverse $A^{\#}$, as well as the Bott-Duffin inverse $A_{(L)}^{(-1)}$ and the generalized Bott-Duffin inverse $A_{(L)}^{(\dagger)}$ can be presented by a unified approach, as generalized inverses $A_{T, S}^{(2)}$ for appropriate choice of matrices $T$ and $S$. For example, the next is valid for a rectangular matrix $A$ (Ben-Israel \& Greville, 2003):

$$
A^{\dagger}=A_{\mathcal{R}\left(A^{T}\right), \mathcal{N}\left(A^{T}\right)}^{(2)}, A_{M, N}^{\dagger}=A_{\mathcal{R}\left(A^{\sharp}\right), \mathcal{N}\left(A^{\sharp}\right)}^{(2)},
$$

where $M, N$ are positive definite matrices of appropriate orders and $A^{\sharp}=N^{-1} A^{T} M$. For a given square matrix $A$ the next identities are satisfied (Ben-Israel \& Greville, 2003; Chen, 1990; Wang et al., 2004):

$$
A^{D}=A_{\mathcal{R}\left(A^{k}\right), \mathcal{N}\left(A^{k}\right)}^{(2)}, k=\operatorname{ind}(A), A^{\#}=A_{\mathcal{R}(A), \mathcal{N}(A)}^{(2)} .
$$

In the next proposition we restate the full-rank representation of $\{2\}$-inverses with prescribed range and null space from (Shen \& Cheng, 2007).

Proposition 1 (Shen \& Cheng, 2007) Let $A \in \mathbb{C}_{r}^{m \times n}, T$ be a subspace of $\mathbb{C}^{n}$ of dimension $s \leq r$ and let $S$ be a subspace of $\mathbb{C}^{m}$ of dimensions $m-s$. In addition, suppose that $W \in \mathbb{C}^{n \times m}$ satisfies $\mathcal{R}(W)=T, \mathcal{N}(W)=S$. Let $W$ has an arbitrary full-rank decomposition, that is $W=F G$. If $A$ has a $\{2\}$-inverse $A_{T, S}^{(2)}$, then:

(1) GAF is an invertible matrix;

(2) $A_{T, S}^{(2)}=F(G A F)^{-1} G$.

\section{Representations Based on QRD and SVD}

In this section, it is assumed that $A \in \mathbb{C}_{r}^{m \times n}$ is an input matrix and $W \in \mathbb{C}_{s}^{n \times m}, 0<s \leq r$ is an arbitrary but fixed matrix. A complete orthogonal factorization of $W$ is defined by

$$
W=U\left[\begin{array}{ll}
T & 0 \\
0 & 0
\end{array}\right] V^{*},
$$


where $T$ is a square nonsingular matrix of dimension $s \times s, s=\operatorname{rank}(W)$. It is assumed that the zero blocks in positions $(1,2)$ and $(2,2)$ of the block matrix in (3) may vanish. In order to simplify computations and derive a full-rank representation of $W$, it is necessary to consider partitions of matrices $U$ and $V$ into appropriate blocks

$$
U=\left[\begin{array}{ll}
U_{s} & U_{R}
\end{array}\right], \quad V=\left[\begin{array}{ll}
V_{s} & V_{R}
\end{array}\right],
$$

where $U_{s}$ and $V_{s}$ denote the first $s$ columns of $U$ and $V$, respectively. Then

$$
W=U_{s} T V_{s}^{*}
$$

is a full-rank factorization of $W$ obtained from the complete orthogonal factorization (3).

In this section we will prove that the $Q R$ factorization and the $S V D$ are various appearances of the complete orthogonal factorization. Also, in Section 4 we show that a complete orthogonal factorization of $W$ can be derived from its reduction to a bidiagonal form.

Suppose that the $Q R$ factorization of $W$ is of the form

$$
W P=Q R
$$

where $P$ is an $m \times m$ permutation matrix, $Q \in \mathbb{C}^{n \times n}, Q^{*} Q=I_{n}$ and $R \in \mathbb{C}_{s}^{n \times m}$ is an upper trapezoidal matrix. Assume that $Q$ and $R$ are partitioned as

$$
Q=\left[\begin{array}{ll}
Q_{S} & Q_{R}
\end{array}\right], \quad R=\left[\begin{array}{cc}
R_{11} & R_{12} \\
O & O
\end{array}\right]=\left[\begin{array}{c}
R_{1} \\
O
\end{array}\right],
$$

where $Q_{s}$ consists of the first $s$ columns of the matrix $Q$ and $R_{11} \in \mathbb{C}^{s \times s}$ is nonsingular.

The idea to calculate the Moore-Penrose inverse using the $Q R$ decomposition is originated in (Katsikis et al., 2011): if $A P=Q R$ is a $Q R$ factorization of $A$, then $A^{\dagger}=P R^{\dagger} Q^{*}$. A generalization of this result to the set of outer inverses is introduced in (Stanimirović et al., 2012b): if $A$ has a $\{2\}$-inverse $A_{\mathcal{R}(W), \mathcal{N}(W)}^{(2)}$, then $R_{1} P^{*} A Q_{s}$ is an invertible matrix and

$$
A_{\mathcal{R}(W), \mathcal{N}(W)}^{(2)}=Q_{s}\left(R_{1} P^{*} A Q_{s}\right)^{-1} R_{1} P^{*}=A_{\mathcal{R}\left(Q_{s}\right), \mathcal{N}\left(R_{1} P^{*}\right)}^{(2)} .
$$

The $Q R$ factorization (6) is not a complete orthogonal factorization unless $R_{12}=0$. Therefore, (7) is not derived from the complete orthogonal factorization. However, $R_{12}$ can be eliminated by applying further orthogonal (or unitary) transformations from the right to the upper trapezoidal matrix $\left[\begin{array}{ll}R_{11} & R_{12}\end{array}\right]$ :

$$
\left[\begin{array}{ll}
R_{11} & R_{12}
\end{array}\right] Z=\left[\begin{array}{ll}
T_{11} & 0
\end{array}\right] \text {. }
$$

This gives the complete orthogonal factorization of $W$ :

$$
W=Q\left[\begin{array}{cc}
T_{11} & 0 \\
0 & 0
\end{array}\right](P Z)^{*}
$$

Therefore, (8) is the complete orthogonal factorization of $W$ arising from (5), where $T$ is equal to $T_{11}$. Furthermore, $W=Q_{s} T_{11}(P Z)^{*}$ is the full-rank factorization of $W$ (called the $Q R$ full-rank factorization). The following representation can be derived as an analogy of (7).

Corollary 1 Let $A \in \mathbb{C}_{r}^{m \times n}$ be the given matrix and $W \in \mathbb{C}_{s}^{n \times m}, s \leq r$ be selected. Assume that (8) the complete orthogonal factorization of $W$. If $A$ has a $\{2\}$-inverse $A_{\mathcal{R}(W), \mathcal{N}(W)}^{(2)}$, then $T_{11}(P Z)^{*} A Q_{s}$ is an invertible matrix and

$$
A_{\mathcal{R}(W), \mathcal{N}(W)}^{(2)}=Q_{s}\left(T_{11}(P Z)^{*} A Q_{s}\right)^{-1} T_{11}(P Z)^{*}=A_{\mathcal{R}\left(Q_{s}\right), \mathcal{N}\left((P Z)^{*}\right)^{*}}^{(2)}
$$

Suppose that the $S V D$ factorization of $W \in \mathbb{C}_{s}^{n \times m}$ is of the general form

$$
W=U \Sigma V^{*},
$$

where $U \in \mathbb{C}_{s}^{n \times n}$ and $V \in \mathbb{C}_{s}^{m \times m}$ are column-orthogonal and $\Sigma \in \mathbb{C}_{s}^{n \times m}$ is a diagonal matrix with the singular values of $W$ in descending order $\sigma_{1} \geq \sigma_{2} \geq \cdots \geq \sigma_{s}$ on the main diagonal. 
The $S V D$ is a complete orthogonal factorization in which the block $T$ is the diagonal matrix with singular values of $W$ on the main diagonal and $U, V$ are left orthogonal. It is known that the $S V D(10)$ can be presented in a more efficient form

$$
W=U_{s} \Sigma_{s} V_{s}^{*}
$$

where

$$
\begin{aligned}
U= & {\left[\begin{array}{ll}
U_{s} & U_{R}
\end{array}\right], V=\left[\begin{array}{ll}
V_{s} & V_{R}
\end{array}\right], \Sigma=\left[\begin{array}{cc}
\Sigma_{s} & O \\
O & O
\end{array}\right], } \\
& U_{s} \in \mathbb{C}^{m \times s}, V_{s} \in \mathbb{C}^{n \times s}, \Sigma_{s}=\operatorname{diag}\left\{\sigma_{1}, \ldots, \sigma_{s}\right\} .
\end{aligned}
$$

Further, discarding small singular values in $\Sigma_{s}$, it is possible to use the Truncated Singular Value Decomposition $(T S V D)$ of $W$ and generate a full-rank factorization of its approximation $W_{(t)}$, defined by

$$
W_{(t)}=U_{t} \Sigma_{t} V_{t}^{*}, t \leq s
$$

Immediately from Proposition and the full rank decomposition (13) we obtain the following representation of outer inverses.

Lemma 1 Let $A \in \mathbb{C}_{r}^{m \times n}$ be the given matrix and $W \in \mathbb{C}_{s}^{n \times m}, s \leq r$ be selected. If (13) is the full-rank factorization of $W_{(t)}$ then the following is valid

$$
A_{\mathcal{N}\left(U_{t}\right), \mathcal{N}\left(V_{t}^{*}\right)}^{(2)}=U_{t}\left(\Sigma_{t} V_{t}^{*} A U_{t}\right)^{-1} \Sigma_{t} V_{t}^{*}, t \leq s .
$$

Algorithm 1 defines the method for computing the outer inverse of $A$ which is based on the $S V D$ full-rank decomposition of $W$.

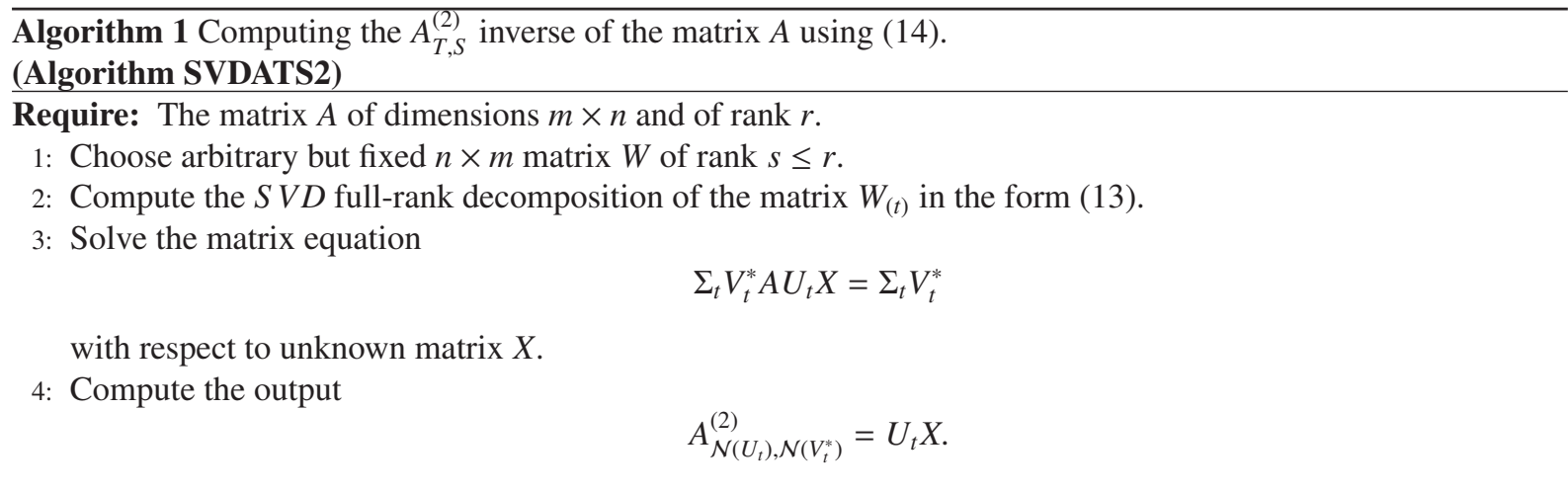

We also define representations based on a combination of the $Q R$ decomposition of $A$ and the $S V D$ of the triangular matrix $R$. This representation is known from (Moor, 1991, 1992). In applications where $m \gg n$, it is often a good idea to use the $S V D$ of the triangular factor that appears in the $Q R$ decomposition of $A$ (see Moor, 1991):

$$
\begin{aligned}
A & =Q R \\
& =Q\left(U^{R} \Sigma^{R}\left(V^{R}\right)^{*}\right) \\
& =\left(Q U_{r}^{R}\right)\left(\Sigma_{r}^{R}\left(V_{r}^{R}\right)^{*}\right),
\end{aligned}
$$

where $U_{r}^{R}$ contains first $r$ columns of $U^{R}, \Sigma_{r}=\operatorname{diag}\left\{\sigma_{1}^{R}, \ldots, \sigma_{r}^{R}\right\}, V_{r}^{R}$ contains first $r$ columns of $V^{R}$.

The full-rank factorization (15) of $A$ gives us idea to apply $S V D$ of the triangular factor arising from the $Q R$ decomposition of $W \in \mathbb{R}_{s}^{n \times m}, 0<s \leq r$ :

$$
W P=Q_{W} R_{W}=Q_{W}\left(U \Sigma V^{*}\right)
$$

Later, one can use the following truncated form of (16):

$$
W_{(t)}=\left(Q_{W} U_{t}\right) \Sigma_{t} V_{t}^{*} P^{*}, 0<t \leq s \leq r,
$$


where $U_{t}$ contains first $t$ columns of $U, \Sigma_{t}=\operatorname{diag}\left\{\sigma_{1}^{R}, \ldots, \sigma_{t}^{R}\right\}$ is a diagonal matrix defined by the first $t$ singular values $\sigma_{1}^{R}, \ldots, \sigma_{t}^{R}$ of $R_{W}$ and $V_{t}$ contains first $t$ columns of $V$. Clearly, (17) is a full-rank factorization of $W_{(t)}$. Therefore, the induced outer inverse of $A$ with the range $\mathcal{R}\left(Q_{W}\right)$ and null space $\mathcal{N}\left(V_{t}^{*} P^{*}\right)$ is defined by

$$
A_{\mathcal{R}\left(Q_{W}\right), \mathcal{N}\left(V_{t}^{*} P^{*}\right)}^{(2)}=\left(Q_{W} U_{t}\right)\left(\Sigma_{t} V_{t}^{*} P^{*} A Q_{W} U_{t}\right)^{-1} \Sigma_{t} V_{t}^{*} P^{*}
$$

Algorithm 2 defines the method for computing outer inverse of $A$ which is defined in (18).

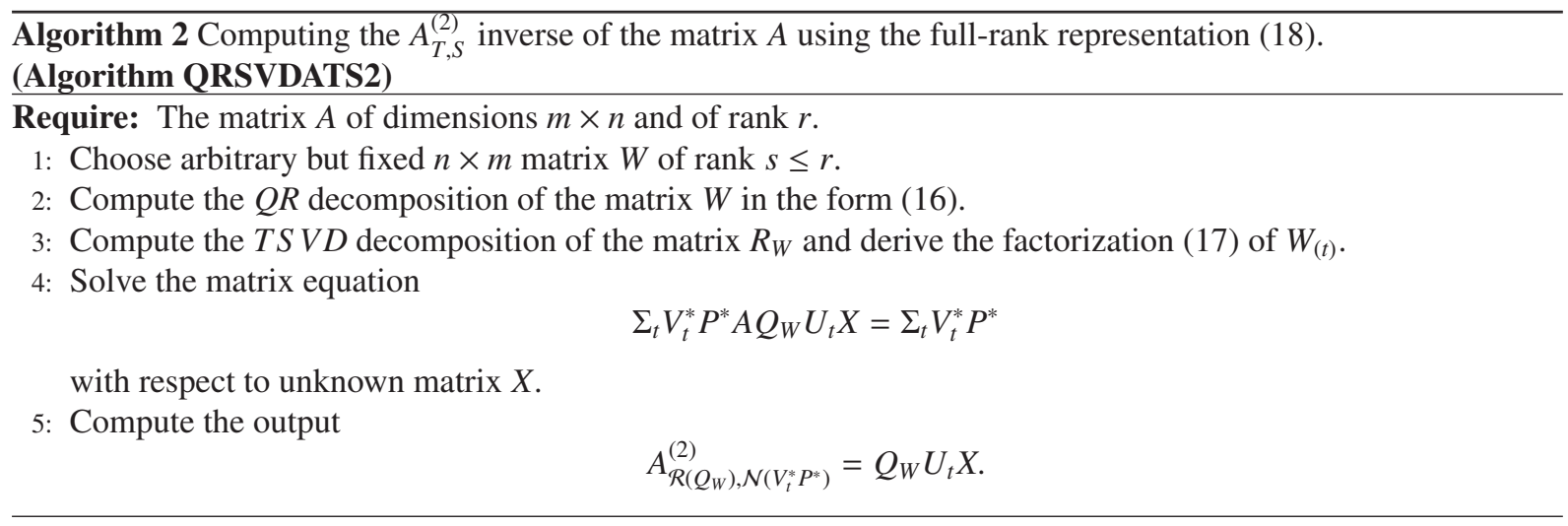

\section{Representations Based on Bidiagonal Forms}

In this section we generalize two algorithms from (Smoktunowicz \& Wróbel, 2012). The first algorithm from (Smoktunowicz \& Wróbel, 2012) is restated in Algorithm 3.

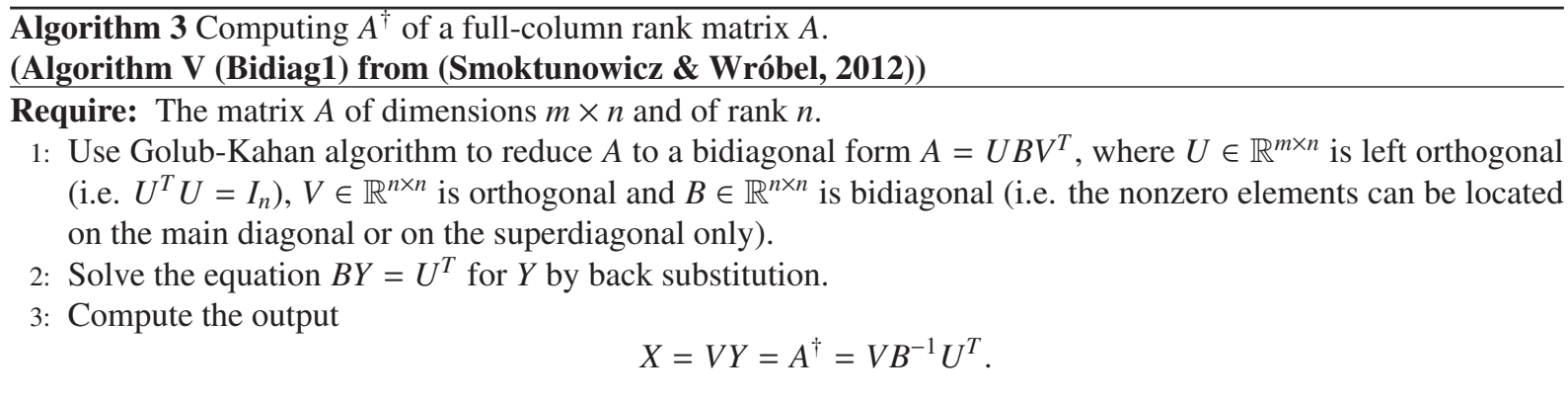

The second algorithm from (Smoktunowicz \& Wróbel, 2012) is restated in Algorithm 4.

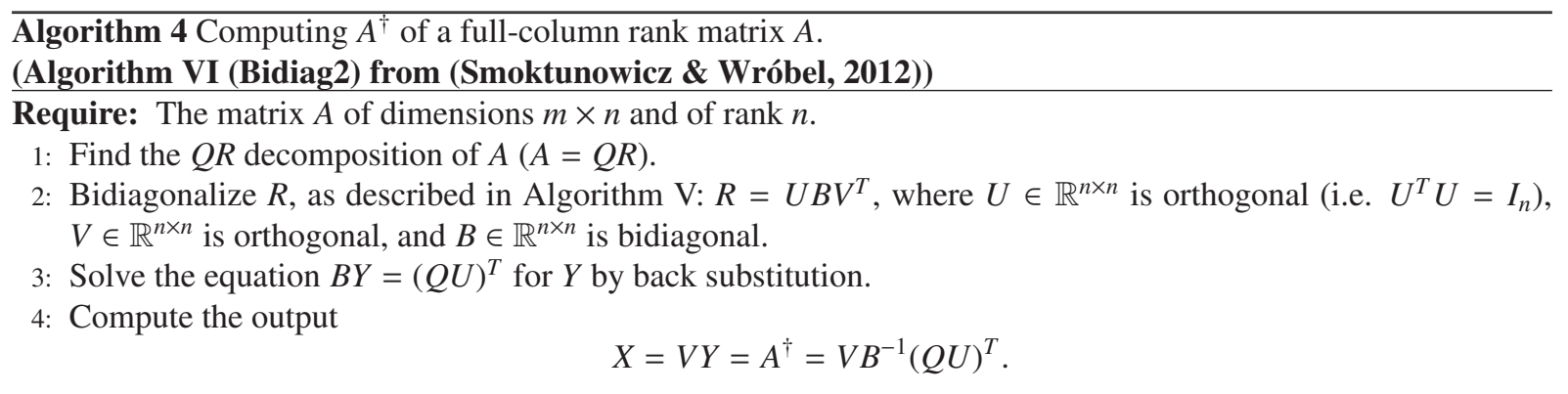

A complete orthogonal factorization of $W$ can be obtained starting from its bidiagonal form. Introduced generalizations of Algorithm 3 and Algorithm 4 are applicable to real full row rank matrices. If $A$ is of the order $m \times n$ and of rank $m \leq n$, introduced algorithms generate the set of outer inverses of $A$ of rank $m$, denoted by $A\{2\}_{m}$. The bidiagonal form of the matrix $W \in \mathbb{R}_{m}^{n \times m}$, defined in (Ralha, 2003), is used to compute outer inverses of $A$. As it is stated in (Smoktunowicz \& Wróbel, 2012), the assumption $\operatorname{rank}(W)=m$ guarantees invertibility of $B$. This means that $W=U B V^{T}$ is a complete orthogonal factorization of $A$. In the sequel we define a method to obtain a complete orthogonal factorization of $W \in \mathbb{R}_{m}^{n \times m}$ starting from its bidiagonal form. 
Proposition 2 (Ralha, 2003) Let $W \in \mathbb{R}^{n \times m}$ satisfy $n \geq m=\operatorname{rank}(W)$. There exist orthogonal $U \in \mathbb{R}^{n \times n}$, orthogonal $V \in \mathbb{R}^{m \times m}$ and upper bidiagonal matrix

$$
B=\left[\begin{array}{c}
\tilde{B} \\
0
\end{array}\right] \in \mathbb{R}^{n \times m},
$$

where the block $\tilde{B}$ is bidiagonal, such that the following factorization of $W$ holds:

$$
W=U B V^{T} \text {. }
$$

Theorem 1 Let $A \in \mathbb{R}_{m}^{m \times n}$ be a given matrix satisfying $n \geq m$ and $W \in \mathbb{R}_{n}^{n \times m}$ be a selected but fixed matrix. Let (19) be the bidiagonal form of $W$. Let $U$ be partitioned into blocks

$$
U=\left[\begin{array}{ll}
U_{m} & U_{R}
\end{array}\right]
$$

where $U_{m}$ denotes the first $m$ rows of $U$. Then

$$
W=U_{m} \tilde{B} V^{T}
$$

is a complete orthogonal factorization of W. Also,

$$
W=F G, \quad F=U_{m}, G=\tilde{B} V^{T}
$$

is a full-rank factorization of Wand

$$
A_{\mathcal{R}\left(U_{m}\right), \mathcal{N}\left(V^{T}\right)}^{(2)}=U_{m}\left(\tilde{B} V^{T} A U_{m}\right)^{-1} \tilde{B} V^{T} .
$$

Using the representation (22) from Theorem we are in a position to state Algorithm 5.

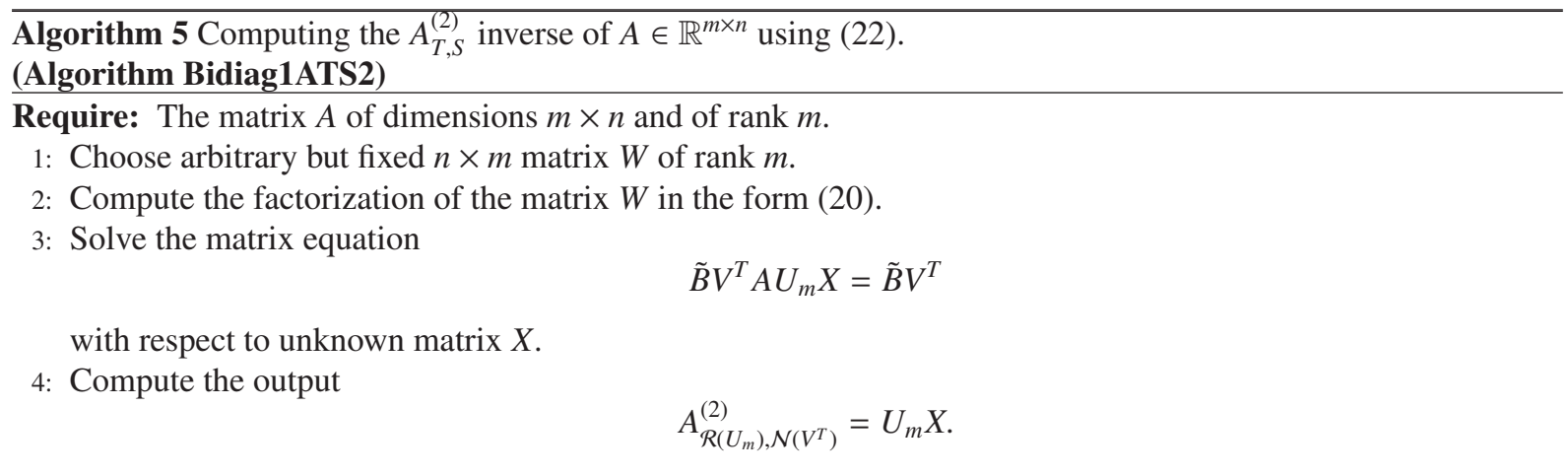

In order to generalize Algorithm 4, in the sequel we generalize the idea of the reduction to bidiagonal form in two stages. If $W \in \mathbb{R}_{m}^{n \times m}$, the generalization is justified in the case when $n$ is much larger than $m$. A $Q R$ decomposition of $W \in \mathbb{R}_{m}^{n \times m}$ is performed in the first stage:

$$
W=Q R=\left[\begin{array}{ll}
Q_{m} & Q_{R}
\end{array}\right]\left[\begin{array}{c}
R_{m} \\
0
\end{array}\right],
$$

where $R_{m} \in \mathbb{R}^{m \times m}$ is upper triangular and $Q_{m}$ denotes the matrix constructed from the first $m$ columns of $Q$. The second stage requires reduction of the relatively small matrix $R_{m}$ into a bidiagonal form

$$
R_{m}=\tilde{U} \tilde{B} \tilde{V}^{T}
$$

All matrices included in the decomposition (24) are of the order $m \times m, \tilde{U}, \tilde{V}$ are orthogonal and $\tilde{B}$ is bidiagonal. Decompositions (23) and (24) imply the following representation of outer inverses of $A$.

Theorem 2 Let $A \in \mathbb{R}_{m}^{m \times n}$ be given and $W \in \mathbb{R}_{m}^{n \times m}$ be chosen matrix. If (23) is a QR decomposition of $W$ and (24) is a bidiagonal form of $R_{m}$, then

$$
A_{\mathcal{R}\left(Q_{m}\right), \mathcal{N}\left(V^{T}\right)}^{(2)}=Q_{m} \tilde{U}\left(\tilde{B} \tilde{V}^{T} A Q_{m} \tilde{U}\right)^{-1} \tilde{B} \tilde{V}^{T}
$$


Proof. We have

$$
\begin{aligned}
W & =\left[\begin{array}{ll}
Q_{m} & Q_{R}
\end{array}\right]\left[\begin{array}{c}
\tilde{U} \tilde{B} \tilde{V}^{T} \\
0
\end{array}\right] \\
& =Q_{m} \tilde{U} \tilde{B} \tilde{V}^{T} .
\end{aligned}
$$

Since

$$
W=F G, \quad F=Q_{m} \tilde{U}, G=\tilde{B} \tilde{V}^{T}
$$

is a full-rank factorization of $W$, the proof immediately follows from the representation of outer inverses with prescribed range and null space from (Shen \& Cheng, 2007).

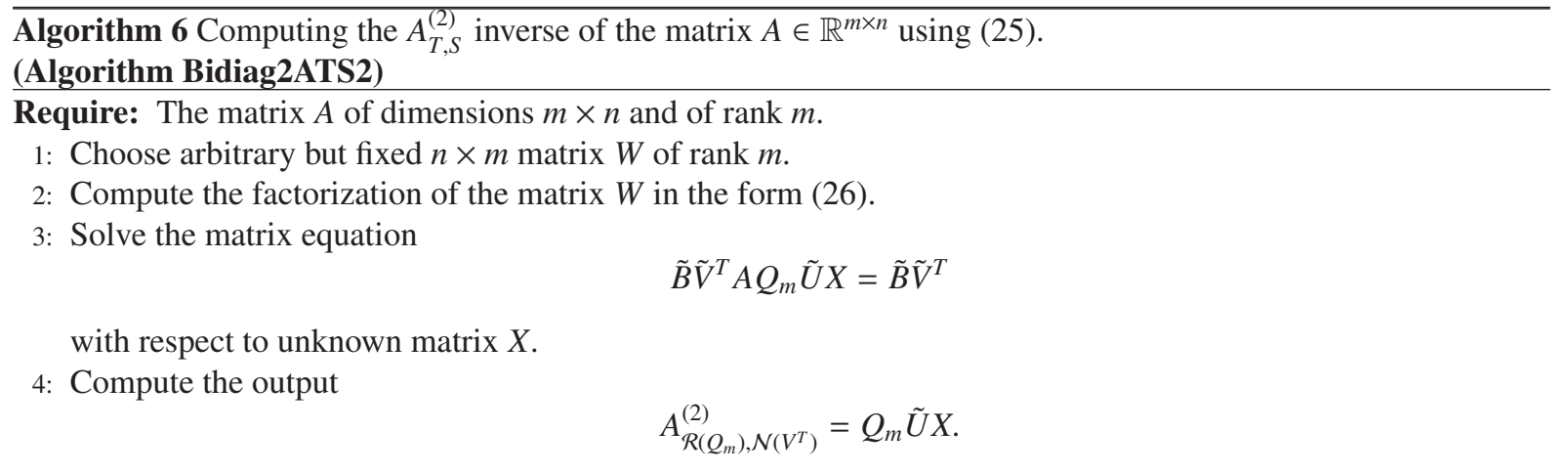

\section{Numerical Experience}

Example 1 In this illustrative example we consider the matrix

$$
A=\left[\begin{array}{lllll}
1 & 2 & 3 & 4 & 1 \\
1 & 3 & 4 & 6 & 2 \\
2 & 3 & 4 & 5 & 3 \\
3 & 4 & 5 & 6 & 4 \\
4 & 5 & 6 & 7 & 6 \\
6 & 6 & 7 & 7 & 8
\end{array}\right]
$$

of rank 4 and choose the matrix

$$
W=\left[\begin{array}{llllll}
13 & 1 & 0 & 0 & 39 & 0 \\
17 & 3 & 0 & 0 & 51 & 0 \\
21 & 4 & 0 & 0 & 63 & 0 \\
25 & 6 & 0 & 0 & 75 & 0 \\
19 & 2 & 0 & 0 & 57 & 0
\end{array}\right]
$$

of rank 2. The $Q R$ decomposition of $W$ is defined by

$$
\{Q, R, P\}=\left\{\left[\begin{array}{cc}
0.299425 & -0.532976 \\
0.391555 & -0.0122351 \\
0.483686 & 0.103891 \\
0.575817 & 0.624632 \\
0.437621 & -0.561096
\end{array}\right],\left[\begin{array}{cccccc}
43.4166 & 7.73898 & 0 . & 0 . & 130.25 & 0 . \\
0 . & 2.47148 & 0 . & 0 . & 7.105 \times 10^{-15} & 0 .
\end{array}\right], P=I_{6}\right\} .
$$

Truncated $S V D$ of the order 2 of the matrix $R$ is defined by the ordered triple

$$
\{U, S, V\}=\left\{\left[\begin{array}{cc}
-0.999999 & 0.00101179 \\
-0.00101179 & -0.999999
\end{array}\right],\left[\begin{array}{cc}
137.513 & 0 . \\
0 . & 2.46756
\end{array}\right],\left[\begin{array}{cc}
-0.315726 & 0.0178024 \\
-0.0562962 & -0.998414 \\
0 . & 0 . \\
0 . & 0 . \\
-0.947179 & 0.0534072 \\
0 . & 0 .
\end{array}\right]\right\}
$$


Outer generalized inverse defined in (18) is equal to

$$
A_{\mathcal{R}\left(Q_{W}\right), \mathcal{N}\left(V_{t}^{*} P^{*}\right)}^{(2)}=\left[\begin{array}{cccccc}
0.0453361 & -0.215651 & 0 . & 0 . & 0.136008 & 0 . \\
0.00990099 & -0.0049505 & 0 . & 0 . & 0.029703 & 0 . \\
0.00364773 & 0.0420358 & 0 . & 0 . & 0.0109432 & 0 . \\
-0.0317874 & 0.252736 & 0 . & 0 . & -0.0953622 & 0 . \\
0.0505472 & -0.227028 & 0 . & 0 . & 0.151641 & 0 .
\end{array}\right] .
$$

In the continuation of this section we will give a series of examples in which we study behavior of introduced representations.

Example 2 In this example we compare algorithms for computing outer inverses based on three different factorizations:

- Algorithm QRATS2 based on the $Q R$ decomposition from (Stanimirović et al., 2012b);

- Algorithm QRSVDATS2 based on the representation (18) and

- Algorithm SVDATS2 based on the SVD from (Shaini \& Hoxha, 2013).

The following code in the programming package Mathematica is applied:

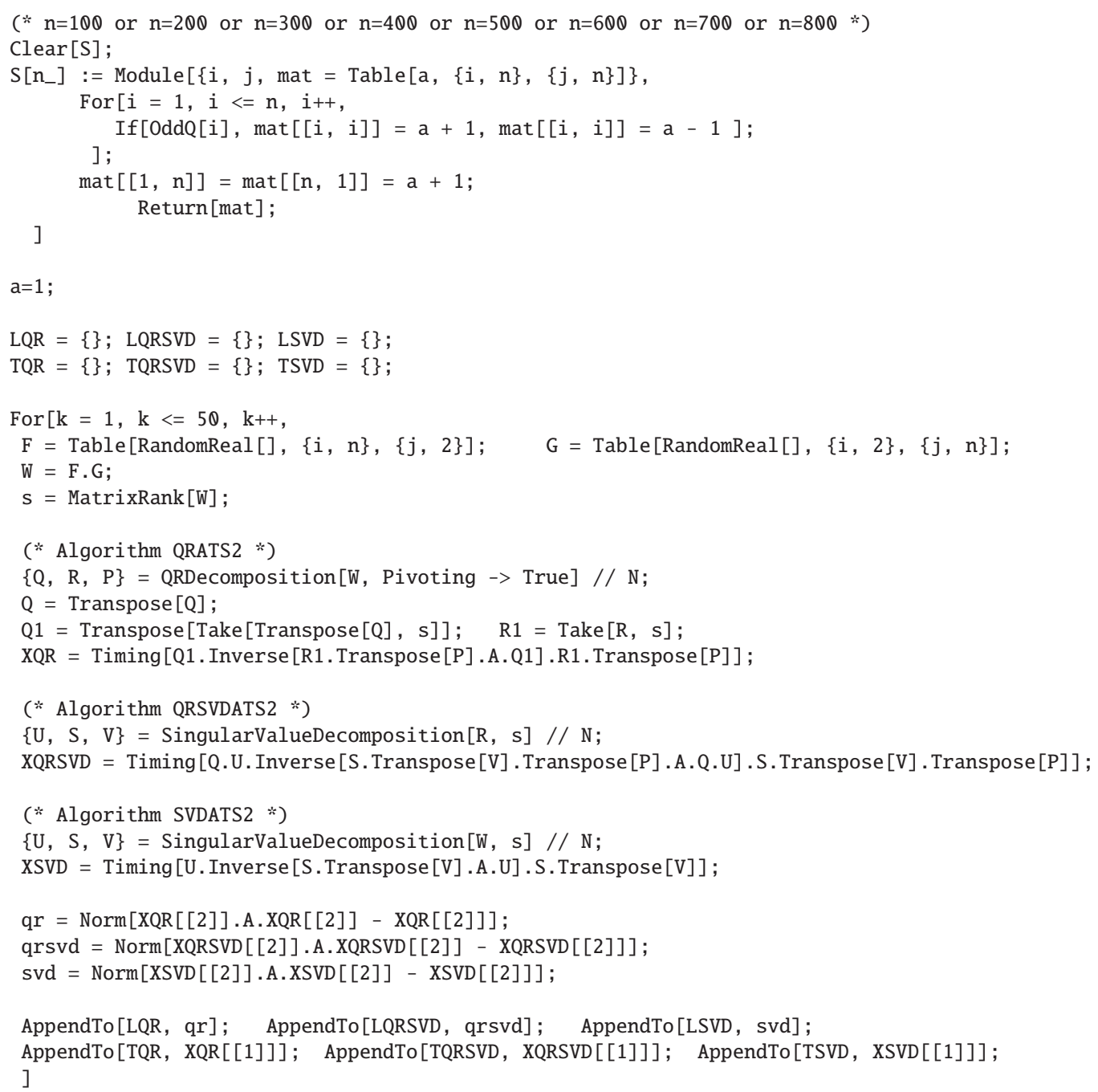

The smallest values in the tables are written in bold font.

In Table 1 and Table 2 we arrange the results obtained applying the above Mathematica code on the test function $S[n]$ from (Zielke, 1986), in the case $a=1$. Dimensions $n$ of test matrices are equal to $n=100,200,300,400,500$, 
$600,700,800$. For each matrix $S[n]$ we generate 50 random matrices $W$ of rank 2 . Two criteria are used for testing the algorithms: precision and CPU time. Let us denote by $X_{k}=\operatorname{QRATS} 2(S[n]), Y_{k}=\operatorname{QRS} \operatorname{VDATS} 2(S[n])$, $Y_{k}=S V D A T S 2(S[n])$ the results generated applying $Q R D$, the combination of $Q R D$ and $S V D$ of $R$ and TSVD for $k$ th generated matrix $W_{k}, k=1, \ldots, 50$. The columns 2,3,4 in Table 1 denote the sum of generated norms:

$$
S Q R_{n}=\sum_{k=1}^{50}\left\|X_{k} A X_{k}-X_{k}\right\|, S Q R S V D_{n}=\sum_{k=1}^{50}\left\|Y_{k} A Y_{k}-Y_{k}\right\|, S S V D_{n}=\sum_{k=1}^{50}\left\|Z_{k} A Z_{k}-Z_{k}\right\| .
$$

Table 1. Comparison of three algorithms for computing outer inverses

\begin{tabular}{|c|c|c|c|}
\hline$n$ & $S Q R_{n}$ & $S Q R S V D_{n}$ & $S S V D_{n}$ \\
\hline 100 & $3.043527549586 \times 10^{-11}$ & $1.454904489449 \times 10^{-11}$ & $1.799685579904 \times 10^{-11}$ \\
\hline 200 & $6.42517329481 \times 10^{-11}$ & $4.418754168002 \times 10^{-11}$ & $4.205730328071 \times 10^{-11}$ \\
\hline 300 & $1.812027062984 \times 10^{-10}$ & $5.292579107393 \times 10^{-10}$ & $1.039752882034 \times 10^{-10}$ \\
\hline 400 & $2.713609669991 \times 10^{-9}$ & $5.292579107393 \times 10^{-10}$ & $5.956789277588 \times 10^{-10}$ \\
\hline 500 & $2.995818463930 \times 10^{-9}$ & $1.314409320131 \times 10^{-9}$ & $1.163914125347 \times 10^{-9}$ \\
\hline 600 & $1.434233650545 \times 10^{-9}$ & $3.91646747789 \times 10^{-10}$ & $3.902297496071 \times 10^{-10}$ \\
\hline 700 & $1.258711792555 \times 10^{-9}$ & $5.1970729464 \times 10^{-10}$ & $4.57213168965 \times 10^{-10}$ \\
\hline 800 & $3.92444897696 \times 10^{-9}$ & $2.728576549705 \times 10^{-9}$ & $4.672371872085 \times 10^{-9}$ \\
\hline
\end{tabular}

Performances of compared methods are tested by using the so-called performance profile, introduced in (Dolan $\&$ Moore, 2002). The underlying performance metric is defined by the accuracy and the CPU time. Following the notations given in the paper (Dolan \& Moore, 2002), the number of solvers is $n_{s}=3$ (the solvers are $S Q R_{n}, S Q R S V D_{n}, S S V D_{n}$ ) and the number of numerical experiments is $n_{p}=8$ (each exploiting 50 randomly matrices $W$ and accordingly generated outer inverses). By $i_{p, s}$ we denote the achieved accuracy obtained by applying the method $s$ on the problem $p$. The quantity

$$
r_{p, s}=\frac{i_{p, s}}{\min \left\{i_{p, s}: s \in\left\{S Q R_{n}, S Q R S V D_{n}, S S V D_{n}\right\}\right\}}
$$

is called the performance ratio. Finally, the performance of the solver $s$ is defined by the following cumulative distribution function

$$
\rho_{s}(\tau)=\frac{1}{n_{p}} \operatorname{size}\left\{p \in \mathcal{P}: r_{p, s} \leq \tau\right\}, s \in\left\{S Q R_{n}, S Q R S V D_{n}, S S V D_{n}\right\}
$$

where $\tau \in \mathbb{R}$ and $\mathcal{P}$ represents the set of problems.

Figure 1 shows the performance profiles for $S Q R_{n}, S Q R S V D_{n}, S S V D_{n}$ regarding the accuracy, using data arranged in Table 1.

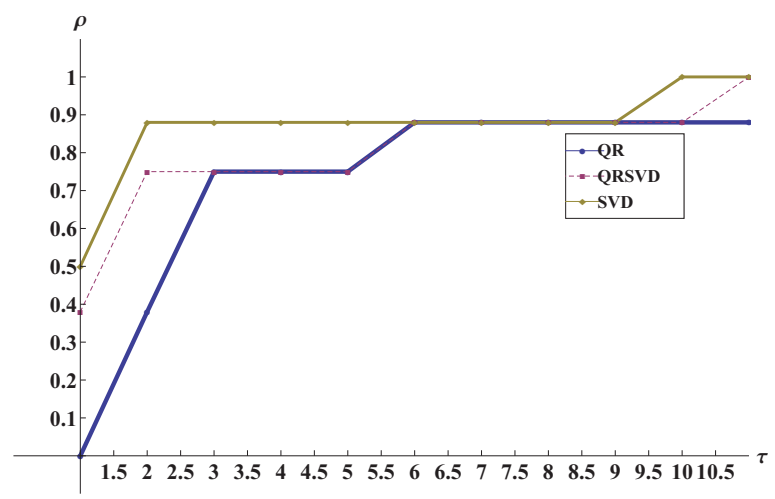

Figure 1. Performance profile regarding the accuracy presented in Table 1

It is observable from Figure 1 that $S S V D_{n}$ and $S Q R S V D_{n}$ methods show better performances compared to $S Q R_{n}$ :

$$
\rho_{S S V D_{n}}(\tau) \geq \rho_{S Q R S V D_{n}}(\tau) \geq \rho_{S Q R_{n}}(\tau), 0 \leq \tau \leq 1
$$


This means that Algorithm $S$ VDATS 2 has the highest probability of being the optimal solver with respect to the numerical accuracy. Also, Algorithm QRS VDATS 2 is better than Algorithm QRATS 2.

Let us denote by $T Q R_{k}, T Q R S V D_{k}, T S V D_{k}$ CPU times spanned by applying $Q R D$, combination of $Q R D$ and $S V D$ of $R$ and $T S V D$ for a selected $n$ and $k$ th generated matrix $W_{k}, k=1, \ldots, 50$. The columns $2,3,4$ in Table 2 denote the sums of CPU times

$$
S T Q R_{n}=\sum_{k=1}^{50} T Q R_{k}, S T Q R S V D_{n}=\sum_{k=1}^{50} T Q R S V D_{k}, S T S V D_{n}=\sum_{k=1}^{50} X T S V D_{k} .
$$

Table 2. CPU time of three algorithms for computing outer inverses

\begin{tabular}{clll}
\hline$n$ & $S T Q R_{n}$ & $S T Q R S V D_{n}$ & $S T S V D_{n}$ \\
\hline 100 & 0.0312002 & $\mathbf{0 . 0 0 0 0 0 0 1}$ & $\mathbf{0 . 0 0 0 0 0 0 1}$ \\
200 & 0.0468003 & $\mathbf{0 . 0 0 0 0 0 0 1}$ & $\mathbf{0 . 0 0 0 0 0 0 1}$ \\
300 & 0.0624004 & $\mathbf{0 . 0 1 5 6 0 0 1}$ & 0.0312002 \\
400 & 0.1248008 & 0.0624004 & $\mathbf{0 . 0 3 1 2 0 0 2}$ \\
500 & 0.202801 & 0.1716011 & $\mathbf{0 . 0 9 3 6 0 0 6}$ \\
600 & 0.3432022 & 0.2028013 & $\mathbf{0 . 1 9 3 6 0 0 6}$ \\
700 & 0.3588023 & 0.3588023 & $\mathbf{0 . 2 3 4 0 0 1 5}$ \\
800 & 0.5304034 & 0.5460035 & $\mathbf{0 . 3 1 2 0 0 2}$ \\
\hline
\end{tabular}

Figure 2 illustrates data arranged in Table 2 and shows the performance profiles for the methods $Q R A T S 2$, QRS VDATS 2, S VDATS 2 regarding the spanned CPU time.

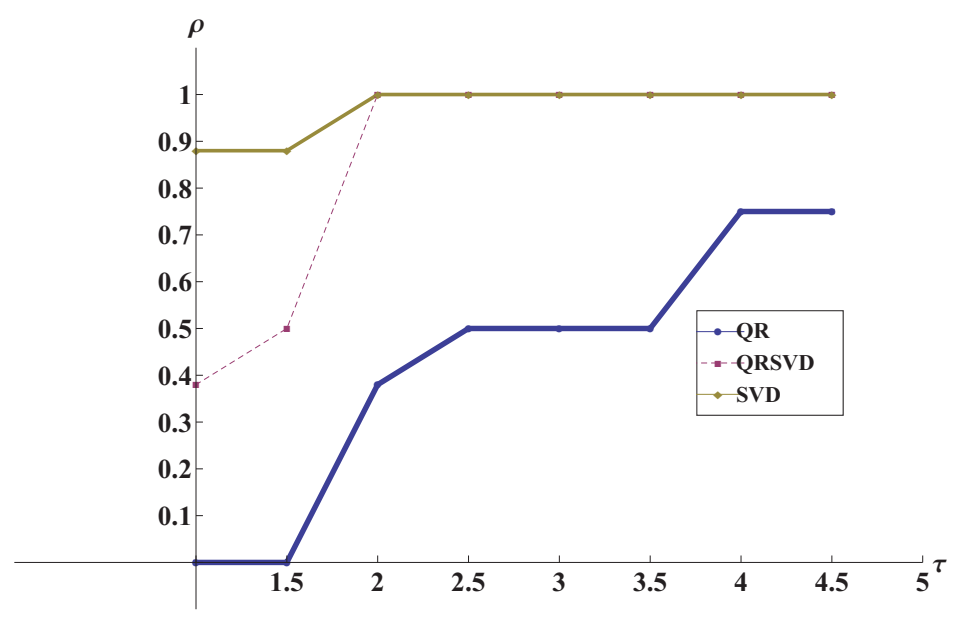

Figure 2. Performance profile regarding the CPU time presented in Table 2

Figure 2 leads to the same conclusion as Figure 1:

$$
\rho_{S T S V D_{n}}(\tau) \geq \rho_{S T Q R S V D_{n}}(\tau) \geq \rho_{S T Q R_{n}}(\tau), 0 \leq \tau \leq 1 .
$$

Example 3 In this example we compare the same algorithms as in Example 2 on the numerical computation of the Moore-Penrose inverse. Instead of randomly generated matrices we use the matrices $\mathrm{W}=$ Transpose $[\mathrm{A}]=$ Transpose $[\mathrm{S}[\mathrm{n}]], n=10,30,50,70,90,110,130,150,170,190$.

The results are arranged in Table 3. Let us denote by

$$
X_{n}=\operatorname{QRATS} 2(S[n]), Y_{n}=\operatorname{QRSVDATS} 2(S[n]), Z_{n}=\operatorname{SVDATS} 2(S[n])
$$

the results generated applying $Q R D$, the combination of $Q R D$ and $S V D$ of $R$ and $T S V D$, respectively. The columns 2, 3, 4 in Table 3 contain the generated matrix norms

$$
Q R_{n}=\left\|X_{n} A X_{n}-X_{n}\right\|, Q R S V D_{n}=\left\|Y_{n} A Y_{n}-Y_{n}\right\|, S V D_{n}=\left\|Z_{n} A Z_{n}-Z_{n}\right\| .
$$


Table 3. Accuracy of three algorithms for computing the Moore-Penrose inverse of $A=S[n]$

\begin{tabular}{|c|c|c|c|}
\hline$n$ & $Q R_{n}$ & $Q R S V D_{n}$ & $S V D_{n}$ \\
\hline 10 & $1.26979128026215 \times 10^{-14}$ & $2.034260922393099 \times 10^{-15}$ & $2.08463132435095 \times 10^{-15}$ \\
\hline 30 & $4.552256280073264 \times 10^{-13}$ & $1.400571444654255 \times 10^{-14}$ & $1.69407576911329 \times 10^{-14}$ \\
\hline 50 & $3.211712544885929 \times 10^{-12}$ & $3.948851248647643 \times 10^{-14}$ & $8.49949726133356 \times 10^{-14}$ \\
\hline 70 & $2.074587304154639 \times 10^{-11}$ & $1.405943038980567 \times 10^{-13}$ & $9.335752912707724 \times 10^{-14}$ \\
\hline 90 & $7.562701917834577 \times 10^{-11}$ & $1.848439361484347 \times 10^{-13}$ & $2.52063399770323 \times 10^{-13}$ \\
\hline 110 & $39340718 \times 10^{-10}$ & 2.995396809 & $4.933328358230868 \times 10^{-13}$ \\
\hline 130 & $7285 \times 10^{-10}$ & 5.76950819 & $8.547396331319857 \times 10^{-13}$ \\
\hline 150 & $9.706992066606753 \times 10^{-10}$ & $6.703513511452163 \times 10^{-13}$ & $1.01164533157711 \times 10^{-12}$ \\
\hline 170 & $62472706 \times 10^{-9}$ & 9.4271119736 & $1.39830112820996 \times 10^{-12}$ \\
\hline 190 & $1.763047487102721 \times 10^{-9}$ & $1.368421169609527 \times 10^{-12}$ & $2.423576221191562 \times 10^{-12}$ \\
\hline
\end{tabular}

From Table 3 it is possible to conclude the following:

1) The method QRATS 2 is the worst solver.

2) The method QRSVDATS 2 reaches the best numerical precision. The only exception is observed in the case $n=70$.

Example 4 In this example we continue comparison of the same algorithms for numerical computation of outer inverse. We again use the matrix $A=S[n]$ and randomly generated matrices $W$ of $\operatorname{rank} \operatorname{rank}(W)=n / 2$. These matrices can be generated by the next Mathematica code:

$\mathrm{F}=$ Table $[$ RandomReal []$,\{\mathrm{i}, \mathrm{n}\},\{\mathrm{j}, \mathrm{n} / 2\}]$

$\mathrm{G}=$ Table $[$ RandomReal []$,\{\mathrm{i}, \mathrm{n} / 2\},\{\mathrm{j}, \mathrm{n}\}]$;

$\mathrm{W}=\mathrm{F} . \mathrm{G}$;

The results are arranged in Table 4. Dimensions $n$ are equal to $n=10,30,50,70,90,110,130,150,170,190$. Let us denote by

$$
X_{n}=\operatorname{QRATS} 2(S[n]), Y_{n}=\operatorname{QRS} \operatorname{VDATS} 2(S[n]), Z_{n}=S \operatorname{VDATS} 2(S[n])
$$

the results generated applying $Q R D$, combination of $Q R D$ and $S V D$ of $R$ and $T S V D$. The columns 2, 3, 4 in Table 3 contain values of the matrix norms

$$
Q R_{n}=\left\|X_{n} A X_{n}-X_{n}\right\|, Q R S V D_{n}=\left\|Y_{n} A Y_{n}-Y_{n}\right\|, S V D_{n}=\left\|Z_{n} A Z_{n}-Z_{n}\right\|
$$

\begin{tabular}{|c|c|c|c|}
\hline$n$ & $Q R_{n}$ & $Q R S V D_{n}$ & $S V D_{n}$ \\
\hline 100 & $6.482337614683532 \times 10^{-11}$ & $2.336597321351282 \times 10^{-12}$ & $2.25369380715732 \times 10^{-12}$ \\
\hline 200 & $1.009070836847863 \times 10^{-10}$ & $4.835373145662078 \times 10^{-12}$ & $9.532052089466464 \times 10^{-12}$ \\
\hline 300 & $1.431377648899222 \times 10^{-9}$ & $7.52993615694554 \times 10^{-11}$ & $2.239950472891654 \times 10^{-10}$ \\
\hline 400 & $8.858839542548144 \times 10^{-9}$ & $2.172761850286787 \times 10^{-10}$ & $2.525893041322895 \times 10^{-10}$ \\
\hline 500 & $6.042211807189602 \times 10^{-9}$ & $3.297464621913326 \times 10^{-10}$ & $3.281406609564898 \times 10^{-10}$ \\
\hline 600 & $2.880744866586528 \times 10^{-7}$ & $1.292875855137494 \times 10^{-8}$ & $1.125846642264049 \times 10^{-8}$ \\
\hline 700 & $2.289567222443336 \times 10^{-6}$ & $1.342219034202508 \times 10^{-8}$ & $1.797521293862002 \times 10^{-8}$ \\
\hline 800 & $4.740735235660559 \times 10^{-8}$ & $1.537611159244433 \times 10^{-9}$ & $1.771785004312878 \times 10^{-9}$ \\
\hline 900 & $1.446198521363777 \times 10^{-8}$ & $5.4865896018928 \times 10^{-10}$ & $6.560027712609556 \times 10^{-10}$ \\
\hline 1000 & $1.433314506856117 \times 10^{-8}$ & $1.10417479414053 \times 10^{-10}$ & $7.640356337951447 \times 10^{-11}$ \\
\hline 2000 & $1.156190659420065 \times 10^{-7}$ & $9.368815439069883 \times 10^{-10}$ & $1.112831852598413 \times 10^{-9}$ \\
\hline 3000 & $3.630671988979119 \times 10^{-7}$ & $7.875340741156123 \times 10^{-10}$ & $1.995284420020749 \times 10^{-9}$ \\
\hline 4000 & 0.00001541881512652355 & $3.562343444447241 \times 10^{-8}$ & $3.05838545101564 \times 10^{-8}$ \\
\hline 5000 & $4.028422642321919 \times 10^{-6}$ & $3.980279026633443 \times 10^{-8}$ & $1.396301973029279 \times 10^{-8}$ \\
\hline
\end{tabular}

Table 4. Accuracy of three algorithms for computing outer inverses of the matrix $A=S[n]$

Comparing the results included in Table 4, it is possible to conclude the following: 
1) The method $Q R A T S 2$ is again the worst solver regarding the numerical precision.

2) The methods QRS VDATS 2 and $S$ VDATS 2 reach much better numerical precision.

Example 5 Finally, the same algorithms are compared in numerical computation of outer inverses of the Lauchli matrix. The Lauchli matrix is a $(n+1) \times n$ matrix defined by (see Higham, 1988)

$$
L(n, \mu)=\left[\begin{array}{cccc}
1 & 1 & \ldots & 1 \\
\mu & & & \\
& \mu & & \\
& & \ddots & \\
& & & \mu
\end{array}\right] .
$$

In this example, the matrix $A$ is equal to $A=L(n, 0.2)$. We again use randomly generated matrices $W$ satisfying $\operatorname{rank}(W)=n / 2$. The results are arranged in Table 5 .

Table 5. Accuracy of three algorithms for computing the outer inverses of the Lauchli matrix

\begin{tabular}{clll}
\hline$n$ & $Q R_{n}$ & $Q R S V D_{n}$ & $S V D_{n}$ \\
\hline 100 & $\mathbf{1 . 0 2 8 6 0 4 5 4 4 2 3 3 8 3 7} \times \mathbf{1 0}^{-11}$ & $1.425460861122351 \times 10^{-11}$ & $1.142795986871279 \times 10^{-11}$ \\
200 & $\mathbf{7 . 1 0 0 0 7 2 5 5 8 5 7 8 7 2 2} \times \mathbf{1 0}^{-12}$ & $1.824218048288609 \times 10^{-11}$ & $1.689039220659226 \times 10^{-11}$ \\
300 & $3.931602647539584 \times 10^{-10}$ & $\mathbf{1 . 5 2 2 8 9 3 4 4 5 4 7 7 7 2 2} \times \mathbf{1 0}^{-10}$ & $3.067952338830401 \times 10^{-10}$ \\
400 & $5.172628286663662 \times 10^{-11}$ & $\mathbf{3 . 5 1 5 5 1 0 6 1 6 2 8 0 8 2 7} \times \mathbf{1 0}^{-11}$ & $4.156475258052759 \times 10^{-11}$ \\
500 & $4.645784213245661 \times 10^{-10}$ & $\mathbf{1 . 3 1 5 5 6 3 8 6 9 3 2 3 6 9 1} \times \mathbf{1 0}^{-10}$ & $3.028534791228661 \times 10^{-10}$ \\
600 & $1.689506089025813 \times 10^{-10}$ & $\mathbf{1 . 0 1 8 1 3 2 7 5 3 1 3 4 3 7 4} \times \mathbf{1 0}^{-10}$ & $1.172500764749819 \times 10^{-10}$ \\
700 & $1.134125813525221 \times 10^{-9}$ & $5.572822660688449 \times 10^{-10}$ & $\mathbf{2 . 5 7 1 5 3 4 3 5 8 9 2 8 7 6 9} \times \mathbf{1 0}^{-\mathbf{9}}$ \\
800 & $5.647648157231927 \times 10^{-10}$ & $\mathbf{1 . 9 4 5 4 8 9 9 7 3 9 7 5 0 6 9} \times \mathbf{1 0}^{-10}$ & $2.76608506543413 \times 10^{-10}$ \\
900 & $4.477591961216335 \times 10^{-10}$ & $4.434485330379887 \times 10^{-10}$ & $\mathbf{2 . 1 2 9 9 8 6 3 2 1 7 5 3 6 2 9} \times \mathbf{1 0}^{-10}$ \\
1000 & $5.175937950315212 \times 10^{-10}$ & $\mathbf{4 . 0 6 6 6 8 3 4 5 2 8 0 7 8 0 5} \times \mathbf{1 0}^{-10}$ & $5.50032373267487 \times 10^{-10}$ \\
2000 & $1.019238815039415 \times 10^{-9}$ & $7.941173235725879 \times 10^{-10}$ & $\mathbf{7 . 0 9 7 2 6 7 0 4 9 8 4 2 3 6} \times \mathbf{1 0}^{-\mathbf{1 0}}$ \\
3000 & $1.803956394814176 \times 10^{-8}$ & $5.854369183636487 \times 10^{-9}$ & $\mathbf{3 . 5 2 2 5 0 2 0 1 6 6 3 8 0 0 2} \times \mathbf{1 0}^{-\mathbf{9}}$ \\
4000 & $4.642390180619101 \times 10^{-9}$ & $4.484612755803374 \times 10^{-9}$ & $\mathbf{3 . 3 4 7 5 6 1 4 8 1 0 8 1 3 5} \times \mathbf{1 0}^{-\mathbf{9}}$ \\
5000 & $2.528918230390071 \times 10^{-6}$ & $4.265161501638872 \times 10^{-6}$ & $\mathbf{6 . 2 3 8 0 0 1 4 6 3 5 4 5 6 4 4} \times \mathbf{1 0}^{-7}$ \\
\hline
\end{tabular}

Comparing the results included in Table 5, it is possible to conclude the following.

1) The method QRATS 2 reaches the minimal precision two times, in the first two cases.

2) The methods $Q R S V D A T S 2$ and $S$ VDATS 2 generate the results with much better numerical precision. Both of them reach the minimal precision six times.

3) QRATS 2 is the best solver for small dimensions $n, Q R S V D A T S 2$ for medium values and SVDATS 2 for greatest dimensions.

Example 6 The matrix $A=L(n, 0.000002)$ is ill-conditioned and causes serious numerical problems in computation of required outer inverses. The results are obtained using randomly generated matrices $W$ of $\operatorname{rank} \operatorname{rank}(W)=n / 2$ and arranged in Table 6. 
Table 6. Accuracy of three algorithms for computing the outer inverses of the ill-conditioned Lauchli matrix

\begin{tabular}{clll}
\hline$n$ & $Q R_{n}$ & $Q R S V D_{n}$ & $S V D_{n}$ \\
\hline 100 & 0.395788193577041 & 0.4493449896897091 & $\mathbf{0 . 1 4 3 0 7 3 9 5 0 0 9 9 1 1 2 7}$ \\
200 & 0.1101693065477999 & $\mathbf{0 . 0 9 0 5 2 4 0 8 7 5 2 2 1 9 6 7 9}$ & 0.1517284556203095 \\
300 & $\mathbf{0 . 5 7 5 7 3 0 4 0 1 9 5 2 1 9 7 9}$ & 0.9945688854731312 & 0.7355659072586593 \\
400 & 0.5008982407371158 & $\mathbf{0 . 2 0 7 1 7 4 5 7 5 9 3 2 6 6 9 3}$ & 0.473611267749282 \\
500 & 0.8474063213377292 & 0.6275335481487543 & $\mathbf{0 . 3 6 4 0 1 3 4 5 2 0 3 0 9 3 1 6}$ \\
600 & 0.3563584901595677 & 0.3442714329338474 & $\mathbf{0 . 1 5 0 3 1 6 6 1 5 2 0 8 6 5 6 6}$ \\
700 & 6.652359033095027 & 2.89843803437463 & $\mathbf{2 . 2 9 9 7 6 5 2 6 1 4 3 5 7 8 4}$ \\
800 & $\mathbf{1 9 . 4 3 1 8 1 2 2 9 0 1 6 7 2 3}$ & 32.86722282380996 & 37.35266633821223 \\
900 & 5.962766800197418 & $\mathbf{3 . 5 5 5 7 7 7 6 4 1 7 2 9 8 6 3}$ & 8.543932380583575 \\
1000 & 14.55693372724122 & $\mathbf{3 . 9 7 3 1 2 8 9 7 1 9 3 3 5 9 8}$ & 5.542061014703714 \\
2000 & 22.84253966856699 & $\mathbf{1 3 . 6 5 7 3 8 0 6 3 3 3 8 7 1 5}$ & 41.93288432714076 \\
3000 & 5.6061607170558 & 62.15805382380937 & $\mathbf{2 1 . 8 5 3 5 4 6 3 2 3 9 9 6 9 3}$ \\
4000 & $\mathbf{2 1 0 . 4 5 2 0 3 6 4 3 9 0 1 2 2}$ & 613.7966082641098 & 721.2117782329316 \\
5000 & 50.09409738330062 & $\mathbf{2 9 . 8 1 1 3 7 0 5 4 7 6 8 0 7 9}$ & 31.69036850919142 \\
\hline
\end{tabular}

The results included in Table 6 show that QRS VDATS 2 is the best solver six times, SVDATS 2 five times and QRATS 2 reaches the best values three times.

\section{Conclusion}

According to performed numerical experiments we conclude the following.

1) The $S V D$ representation of outer inverses with prescribed range and null space is the most efficient in the case of low rank matrices $W$.

2) The combination of $Q R$ and $S V D$ decomposition of outer inverses with prescribed range and null space is the most efficient in the case $W=A^{T}$.

3) The combination of $Q R$ decomposition and $S V D$ is a significant improvement of the $Q R$ decomposition method from (Stanimirović et al., 2012b).

Also, in the present paper we show that the factorizations based on a bidiagonal form are useful in representation of outer inverses of matrices with full column rank.

\section{References}

Ben-Israel, A., \& Greville, T. N. E. (2003). Generalized inverses: Theory and Applications (2nd ed.). Springer.

Chen, Y. (1990). The generalized Bott-Duffin inverse and its application. Linear Algebra Appl., 134, 71-91. http://dx.doi.org/10.1016/0024-3795(90)90007-Y

De Moor, B. (1991). Generalizations of the singular value and QR decompositions. Signal Processing, 25, 135146.

De Moor, B., \& Dooren, P. V. (1992). Generalizations of the singular value and QR decompositions. SIAM J. Matrix Anal. Appl., 13, 993-1014.

Dolan, E. D., \& Moré, J. J. (2002). Benchmarking optimization software with performance profiles. Math. Program., 91, 201-213. http://dx.doi.org/10.1007/s101070100263

Getson, A. J., \& Hsuan, F. C. (1988). \{2\}-Inverses and their Statistical Applications. Lecture Notes in Statistics, 47. Berlin: Springer.

Goodall, C. R. (1993). Computation Using the QR Decomposition. In C. R. Rao (Ed.), Handbook of Statistics (Vol. 9, pp. 467-508).

Higham, N. J. (n.d.). The Matrix Computation Toolbox. Retrieved from http://www.ma.man.ac.uk/ higham/mctoolbox

Husen, F., Langenberg, P., \& Getson, A. (1985). The $\{2\}$-inverse with applications to statistics. Linear Algebra Appl., 70, 241-248. http://dx.doi.org/10.1016/0024-3795(85)90055-2

Issa, A. S., \& Bdair, O. M. (2014). Some Bounds of the Zeros of Polynomials Based on the QR-Decomposition 
and the LU-Decomposition of the Frobenius Companion Matrix. Journal of Mathematics Research, 6 33-41. http://dx.doi.org/10.5539/jmr.v6n1p33

Katsikis, V. N., Pappas, D., \& Petralias, A. (2011). An improved method for the computation of the Moore-Penrose inverse matrix. Appl. Math. Comput., 217, 9828-9834. http://dx.doi.org/10.1016/j.amc.2011.04.080

Malik, S. B., \& Thome, N. (2014). On a new generalized inverse for matrices of an arbitrary index. Appl. Math. Comput., 226, 575-580. http://dx.doi.org/10.1016/j.amc.2013.10.060

Nashed, M. Z. (1976). Generalized Inverse and Applications. New York: Academic Press.

Nashed, M. Z., \& Chen, X. (1993). Convergence of Newton-like methods for singular operator equations using outer inverses. Numer. Math., 66, 235-257.

Ralha, R. (2003). One-sided reduction to bidiagonal form. Linear Algebra Appl., 358, $219-238$. http://dx.doi.org/10.1016/S0024-3795

Shaini, B. I., \& Hoxha, F. (2013). Computing generalized inverses using matrix factorizations. Facta Universitatis (Niš), Ser. Math. Inform., 28, 335-351.

Sheng, X., \& Chen, G. (2007). Full-rank representation of generalized inverse $A_{T, S}^{(2)}$ and its applications. Comput. Math. Appl., 54, 1422-1430. http://dx.doi.org/10.1016/j.camwa.2007.05.011

Smoktunowicz, A., \& Wróbel, I. (2012). Numerical aspects of computing the Moore-Penrose inverse of full column rank matrices. BIT Numer. Math., 52, 503-524. http://dx.doi.org/10.1007/s10543-011-0362-0

Stanimirović, P. S., Pappas, D., Katsikis, V. N., \& Stanimirović, I. P. (2012a). Computation of $A_{T, S}^{(2)}$-inverses using QDR factorization. Linear Algebra Appl., 437 1317-1331. http://dx.doi.org/10.1016/j.laa.2012.04.026

Stanimirović, P. S., Pappas, D., Katsikis, V. N., \& Stanimirović, I. P. (2012b). Full-rank representations of outer inverses based on the QR decomposition. Appl. Math. Comput., 218, 10321-10333. http://dx.doi.org/10.1016/j.amc.2012.04.011

Wang, G., Wei, Y., \& Qiao, S. (2004). Generalized Inverses: Theory and Computations. Beijing/New York: Science Press.

Watkins, D. (2002). Fundamentals of Matrix Computations. New York: Wiley-Interscience.

Zheng, B., \& Bapat, R. B. (2004). Generalized inverse $A_{T, S}^{(2)}$ and a rank equation. Appl. Math. Comput., 155(2), 407-415. http://dx.doi.org/10.1016/S0096-3003(03)00786-0

Zielke, G. (1986). Report on test matrices for generalized inverses. Computing, 36, $105-162$. http://dx.doi.org/10.1007/BF02238196

\section{Copyrights}

Copyright for this article is retained by the author(s), with first publication rights granted to the journal.

This is an open-access article distributed under the terms and conditions of the Creative Commons Attribution license (http://creativecommons.org/licenses/by/3.0/). 